\title{
Near infrared observations of the truncation of stellar disks
}

\author{
E. Florido ${ }^{1}$, E. Battaner ${ }^{1}$, A. Guijarro ${ }^{1}$, F. Garzón ${ }^{2}$, and J. Jiménez-Vicente ${ }^{3}$ \\ 1 Dpto. Física Teórica y del Cosmos, Universidad de Granada, Spain \\ 2 Instituto de Astrofísica de Canarias, Vía Láctea, s/n, La Laguna, Tenerife \\ 3 Groningen Kapteyn Laboratorium, Groningen, The Netherlands
}

Received 20 April 2001 / Accepted 22 August 2001

\begin{abstract}
We present a first study of truncation of the stellar disks of spiral galaxies in the near infrared. Observations of NGC 4013, NGC 4217, NGC 6504 and NGC 5981 were made with the CAIN NIR camera on the CST in Tenerife. This wavelength range provides the best description of the phenomenon, not only because extinction effects are minimized, but also because the distribution of the old stellar population is directly obtained. The four galaxies are edge-on and an inversion method was developed to obtain the deprojected profiles. We did not assume any model of the different galactic components. The "truncation curve", i.e. $T(R)=\mu(R)-\mu_{\mathrm{D}}(R)$, where $\mu$ is the actual surface brightness in mag/ $\operatorname{arcsec}^{2}$ and $\mu_{\mathrm{D}}$ the exponential disk surface brightness, has been obtained with unprecedented precision. It is suggested that $T(R)$ is proportional to $\left(R_{\mathrm{t}}-R\right)^{-1}$, where $R_{\mathrm{t}}$ is the truncation radius, i.e. the radius beyond which no star is observed.
\end{abstract}

Key words. galaxies: structure, photometry

\section{Introduction}

At large radii the stellar density of disks decreases faster than an exponential until reaching a cut-off or truncation radius $R_{\mathrm{t}}$, where it vanishes. This morphological feature was discovered by van der Kruit (1979) and later studied in more detail by van der Kruit \& Searle (1981a,b, 1982). Recently, this phenomenon has been reconsidered by means of samples larger than the seven edge-on galaxies observed by van der Kruit and Searle and by improved observational techniques. Barteldrees \& Dettmar (1994), Pohlen et al. (2000), Pohlen et al. (2000) and de Grijs et al. (2001) have provided the basic information about truncations in the optical range for external galaxies. Our Galaxy also presents a truncation, although it is more difficult to observe (Habing 1988; Robin et al. 1992; Ruphy et al. 1996; Freudenreich 1998). Porcel et al. (1997) found that the Milky Way cut-off radius cannot be placed at distances larger than $15 \mathrm{kpc}$. Truncations of stellar disks have been reviewed by van der Kruit (2000). Much remains to be done both from theory and observations to understand this phenomenon.

\section{A) Lack of theoretical explanation}

The above studies have established the universality of the phenomenon. Most galaxies, if not all, seem to have truncated stellar disks, sensitivity limits alone are unable

Send offprint requests to: E. Florido, e-mail: estrella@ugr.es to explain this feature. This fact emphasizes the theoretical importance of the topic. However, truncations constitute one of the most important challenges in galactic dynamics. Though several hypotheses have been considered, this phenomenon remains completely unexplained.

It was suggested by van der Kruit (2000) that stellar truncation is accompanied by a significant drop in rotation velocity, with NGC 4013, NGC 891 and NGC 5907 being clear examples of this. If this fact is confirmed, and actually takes place in most truncated disks, it would mean that there is a true decrease in the radial distribution of the total density, i.e. the sum of both, the gas and the stellar densities. Theories suggesting that stellar truncation is due to a cut-off of the star formation rate beyond a certain radius should be reconsidered, as in this case the total gas plus star density would not present any discontinuity. The confirmation of a drop in rotation velocity close to the stellar truncation would pose serious difficulties for the most promising hypothesis, maintained by Kennicutt (1989) and others, in which star formation does not proceed when the gas density is lower than a certain threshold value and would reject all theories in which stars do not exist beyond the truncation radius, because they are not formed.

Another argument against the absence of star formation as the cause of stellar truncation is that we do see star formation beyond the truncation radius of the 
Milky Way. Molecular clouds are often associated with HII regions, IRAS sources, $\mathrm{H}_{2} \mathrm{O}$ masers and other objects characterizing the presence of the formation of high mass stars (Mead et al. 1987; Mead et al. 1990; Brand \& Wouterloot 1994; Rudolph et al. 1996; Williams \& McKee 1997; May et al. 1997; Kobayashi \& Tokunaga 2000, and others). A high star formation rate is also observed in other galaxies (Lequeux \& Guelin 1996). Wouterloot et al. (1988) and Ferguson et al. (1998) found the important result that the amount of star formation per unit mass of $\mathrm{H}_{2}$ at $R=15 \mathrm{kpc}$ is equal to that in the solar neighbourhood. The ratio $N(\mathrm{HII}) / \sigma\left(\mathrm{H}_{2}\right)$ at $R=15 \mathrm{kpc}$ was found to be higher (by a factor 10/7) than in the solar neighbourhood. Though some differences are found between the outer galaxy molecular clouds and the inner ones at $\sim R_{\odot}$, they have much in common, such as a similar star formation efficiency (Santos et al. 2000) and kinetic temperature (Brand \& Wouterloot 1996). The similarities are more noticeable if we compare molecular clouds at $R>R_{\mathrm{t}}$ (where $R_{\mathrm{t}}$ is the truncation radius) and $R<R_{\mathrm{t}}$ but close to $R_{\mathrm{t}}$. This was done by Brand \& Wouterloot (1991, 1994) and Wouterloot et al. (1993) with their sample for $16 \mathrm{kpc}<R<20 \mathrm{kpc}$ and the sample by Mead \& Kutner (1988) for $R \sim 13 \mathrm{kpc}$. More information about molecular clouds beyond the solar radius has been provided by Brand \& Wouterloot (1995), Wouterloot \& Brand (1996) and Wouterloot et al. $(1995,1997)$. The range of masses and sizes are very similar, and hence the densities should be similar. Cloud formation could be much more inefficient than at smaller $R$ (Brand \& Wouterloot 1991). There is indeed, a sharp decrease in $\mathrm{H}_{2}$, but not that pronounced in $\mathrm{HI}$ gas, which might suggest that the formation of molecular clouds out of HI gas is not as efficient beyond some radius. Small unobservable clouds could have no star forming capacity. However, the sudden step of the rotation curve mentioned by van der Kruit (2000) remains unexplained.

A simplified but reasonable picture would then be: the amount of molecular hydrogen and the number of clouds decrease; but the density within a cloud remains more or less constant; therefore, if there is a minimum $\mathrm{H}_{2}$ density for star formation it cannot explain the truncation of the stellar disk.

Then the puzzling question is: if there is star formation beyond $R_{\mathrm{t}}$, where are the stars? There are two possible answers: a) star formation at these large radii is a recent or transient process, so that stars have not been continuously filling this region. Suppose, for instance, that the outer disk has been formed recently, because the disk forms slowly and its radius increases over time. This hypothesis is considered as a possibility by de Grijs et al. (2000) and van der Kruit (2000) and has some theoretical support from early works by Larson (1976) and Gunn (1982). Given our present uncertainties about disk formation, though, this hypothesis is rather speculative. b) stars, once born, then migrate away. This could be the case if stars and gas have different dynamical behaviours, being subject to different forces. Newly formed stars could be subject to other forces and migrate from their birth place.

B) Observational problems

There are two basic features in the surveys and analysis carried out until now which are improved in this work. First, previous observational studies have been made at optical wavelengths, and therefore extinction introduces a severe limitation on the interpretation of the results. Second, the analysis is usually based upon a specific galaxy model with various components of which the space distribution is specified by means of a number of free parameters, which are determined by fitting the observations. However, with this procedure, what is obtained is, in part, what is assumed. Mathematical expressions are still insufficient for many galaxy components.

This fact is specially problematic in the truncation region. It is known that the truncation is not completely sharp, but rather starts as a smooth deviation of the "exponential" disk (i.e. linear when using $\mu$ ). A truncation curve $T(R)$ would quantify this smooth deviation and can be defined precisely as $T(R)=\mu(R)-\mu_{\mathrm{D}}(R)$ where $\mu(R)$ is the observed surface brightness in mag/arcsec ${ }^{2}$ and $\mu_{\mathrm{D}}(R)$ is the exponential surface brightness extrapolated from the inner disk. We know $T\left(R_{\mathrm{t}}\right)=\infty$ in units of $\mathrm{mag} / \operatorname{arcsec}^{2}$, where $R_{\mathrm{t}}$ is the truncation radius. Previous analysis have mainly considered $R_{\mathrm{t}}$. Truncations are an interesting object of study, as they could reveal the historical and dynamical properties of a galaxy. However the whole truncation curve, $T(R)$ also contains valuable information. It is therefore worrying that the mathematical expression of $T(R)$ was assumed rather than obtained as a chief objective.

To avoid the extinction deformation of the radial profiles, we have observed in the near infrared, so we are mostly dealing with the old stellar population. We present observations in $J$ and $K$ s. Extinction in $J$ is more severe than it is in $K$ s. Therefore, conclusions obtained from our measurements in $K$ s are more reliable.

Complementary studies in other colours has been addressed by the above cited texts. NIR CCD-like arrays already exist some years, but the recent improvement of two-dimension detectors and, in particular, that of CAIN, has made it possible to reach the truncation region.

To avoid model-dependent results, we have used a numerical inversion method. Binney \& Tremaine (1987) describe another analytic method to carry out this deprojection, based on the Abell integral. In our procedure, however, only two assumptions are necessary: axisymmetry and negligible extinction. These two conditions are by no means guaranteed in a disk galaxy but it should be taken into account that axisymmetry is implicitly assumed in other procedures. Moreover, we have two sides in an edge-on galaxy, which are all very similar in our sample. Even if a non-axisymmetric disk could exhibit two similar sides when a galaxy is seen edge-on, this is rather improbable. Extinction is a problem when using methods based on previous modeling, as dust often has a ring structure rather than following an exponential law and the dust 

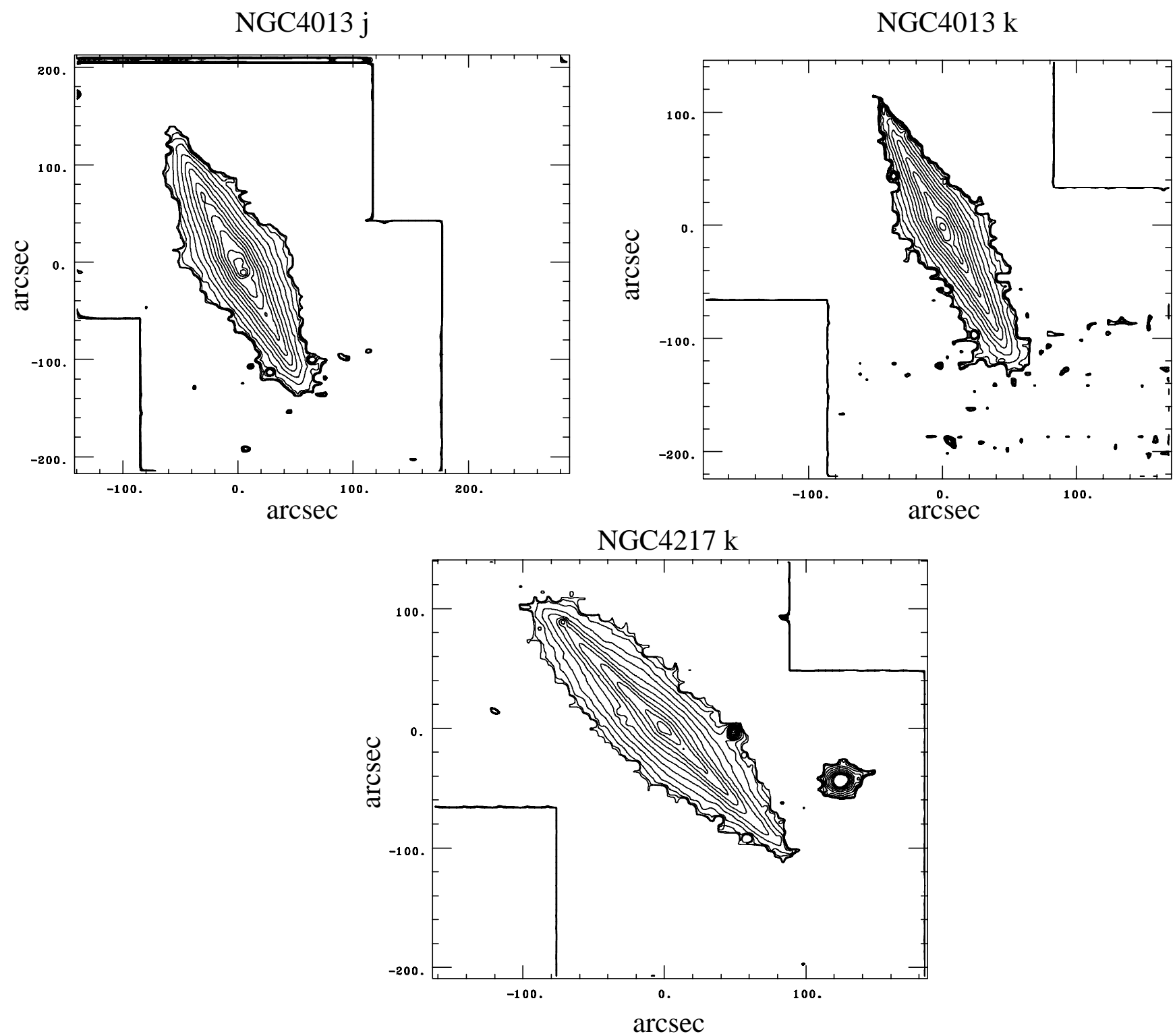

Fig. 1. Contour maps for the observed galaxies: The interval between isophotes is 0.5 magnitudes/arcsec ${ }^{2}$ in all maps. The lower value is 15 magnitudes/ $\operatorname{arcsec}^{2}$ for NGC 5981, NGC 4013 in $K \mathrm{~s}$, NGC 6504 in $K$ s and NGC 6504 in $H$; 15.5 mag/arcsec ${ }^{2}$ for NGC 6504 in $J$ and NGC 4217 in Ks and 16 for NGC 4013 in $J$. East is at bottom and North on right.

Table 1. Observational parameters for the galaxies.

\begin{tabular}{|rccccc|}
\hline Galaxy & Day & Passband & $\begin{array}{c}\text { Exp. time } \\
\text { object+sky(m) }\end{array}$ & Seeing & $\begin{array}{c}3 \sigma \text { level } \\
\text { mag/arcsec }\end{array}$ \\
\hline NGC 4013 17,19 & $K \mathrm{~s}$ & 96 & 1.94 & 20.6 \\
& 13,17 & $J$ & 80 & 1.80 & 22.0 \\
NGC 4217 & 16 & $K \mathrm{~s}$ & 96 & 1.27 & 20.4 \\
& 18 & $\mathrm{~J}$ & 80 & 1.39 & 21.8 \\
& & & & & \\
NGC 5981 & 20 & $K \mathrm{~s}$ & 64 & 1.45 & 19.9 \\
& & & & & \\
NGC 6504 & 16 & $K \mathrm{~s}$ & 88 & 1.27 & 20.4 \\
& 15 & $J$ & 64 & 2.96 & 21.5 \\
& 18 & $H$ & 64 & 1.57 & 20.5 \\
\hline
\end{tabular}

Table 2. Physical parameters for the observed galaxies obtained from LEDA database (http://leda.univ-lyon1.fr): RA and DEC are the right ascension and declination in 2000, $\mathrm{PA}$ is the position angle, $d$ the distance and mabs the absolute $B$-magnitude.

\begin{tabular}{|c|c|c|c|c|c|c|}
\hline Galaxy & $\begin{array}{c}\text { RA } \\
(\mathrm{h} \mathrm{m} \mathrm{s})\end{array}$ & $\begin{array}{c}\text { Dec } \\
\left(\mathrm{o}^{\prime \prime}{ }^{\prime \prime}\right)\end{array}$ & Type & $\begin{array}{c}\text { PA } \\
\mathrm{o}\end{array}$ & $\begin{array}{c}\mathrm{d} \\
(\mathrm{Mpc})\end{array}$ & $m_{\mathrm{abs}}$ \\
\hline NGC 4013 & 115831.5 & 435651 & $\mathrm{Sb}$ & 66 & 11.16 & -19.47 \\
NGC 4217 & 121550.8 & 470530.8 & $\mathrm{Sb}$ & 50 & 13.64 & -19.96 \\
NGC 5981 & 153753.5 & 592328.7 & $\mathrm{Sbc}$ & 140 & 30.18 & -20.34 \\
NGC 6504 & 17565.7 & 331231.7 & Sbc & 94 & 63.18 & -22.28 \\
\hline
\end{tabular}



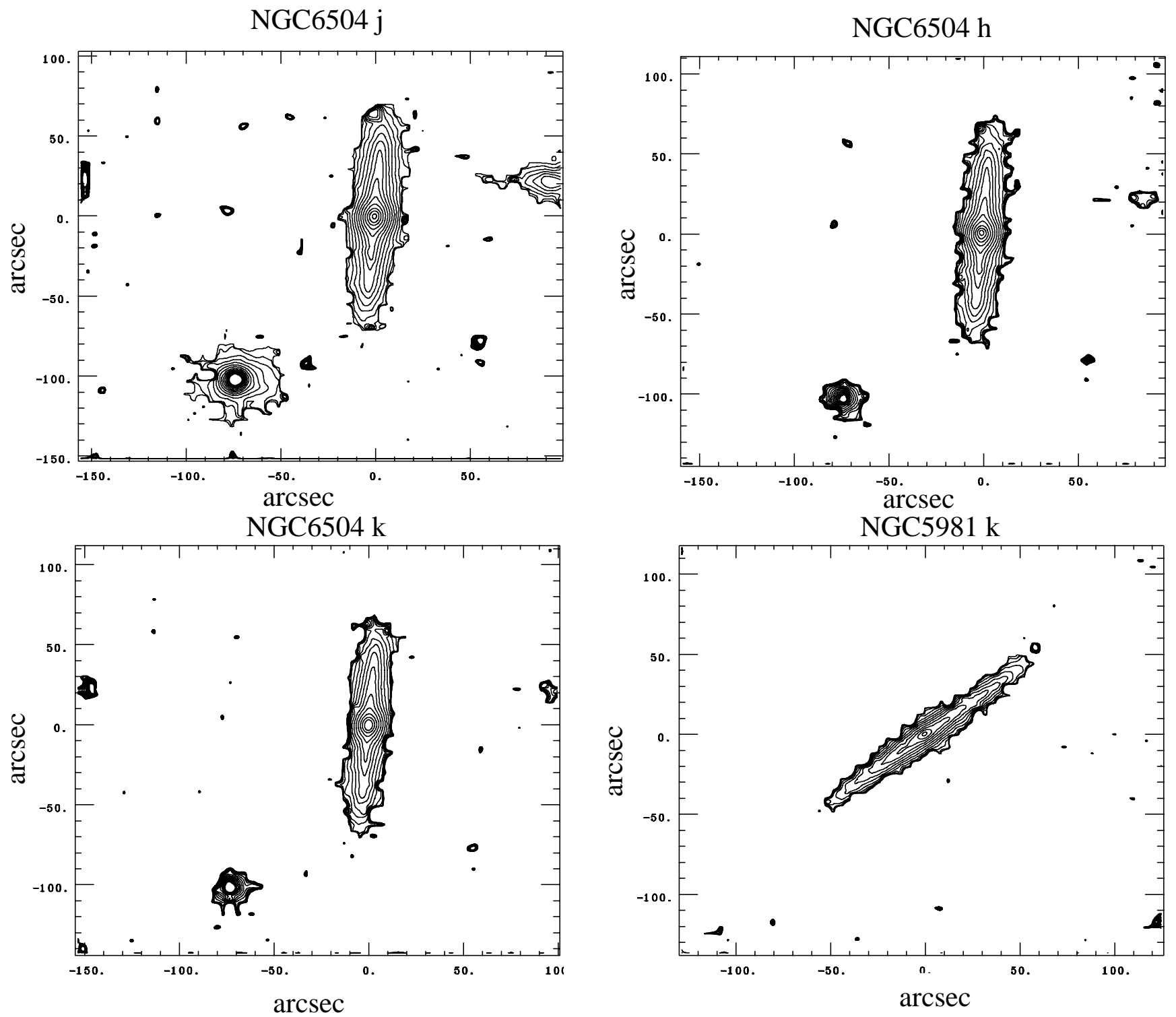

Fig. 1. continued.

distribution must be risky prescribed. Dealing with NIR observations and observing that our $\mu(z)$-profiles do not show a secondary minimum produced by a dust lane, inspires confidence in our method. Also, extinction is probably no longer important in the peripheral truncation region.

With this small number of assumptions required, we obtain a non-model-dependent deprojection.

\section{Observations and reduction}

The observations were carried out at the $1.5 \mathrm{~m}$ CST in the Teide Observatory, Tenerife, with the NIR camera CAIN. This is a common user 2D NIR image camera equipped with a $256^{2}$ NICMOS detector array. Two different plate scales (0.4 and 1.0 arcsec/pixel) are selectable to obtain a narrow or wide field image. We used the wide field optics, which has an effective field of view of $4.3 \times 4.3 \mathrm{arcmin}$. The objects were selected according to their projected size $\left(D_{25}\right)$ to fit within that FOV. The detector control and read-out system was, at the time of the observations, based on dedicated transputer design electronics. In June 1999, the electronics was upgraded to a new design based on San Diego State University controller, adapted in house to the NIR, which provides better noise figures and stability. The transputer controller exhibits several noise correlated patterns which have to be removed during the reduction process by the use of specifically designed software routines.

The observed galaxies were the edge-on galaxies NGC 4013, NGC 4217, NGC 6504 and NGC 5981. They were observed in the period 13-19 April 1999, as shown in Table 1. The basic physical parameters of these galaxies are shown in Table 2.

In order to correct for the bright and rapidly varying NIR sky background, the telescope was alternatively pointed to six fields in the sky in the order 
$\mathrm{O}_{1} \mathrm{~S}_{1} \mathrm{~S}_{2} \mathrm{O}_{2} \mathrm{O}_{1} \mathrm{~S}_{3} \mathrm{~S}_{4} \mathrm{O}_{2}$, where the S's are background and the O's contain the galaxy. The $\mathrm{O}_{2}$ field was offset with respect to $\mathrm{O}_{1}, 15^{\prime \prime} \mathrm{N}$ and $15^{\prime \prime} \mathrm{E}$. $\mathrm{S}_{1}$ was $600^{\prime \prime} \mathrm{W}$ from $\mathrm{O}_{1} ; \mathrm{S}_{2}, 900^{\prime \prime} \mathrm{W} ; \mathrm{S}_{3}, 600^{\prime \prime} \mathrm{E}$ from $\mathrm{O}_{2} ; \mathrm{S}_{4}, 900^{\prime \prime} \mathrm{W}$. Each exposure lasted about 2 min.

It was very important to perform good flat fielding, sky subtracting and mosaicing. We used the data reduction package developed by R. Peletier, REDUCE, within IRAF, which is specially suitable for data with a large sky background. We took object images, bias frames at the beginning and/or at the end of the night, dark frames for the two exposure times used (10 and $30 \mathrm{~s}$ ) and flatfields to calibrate the sensitivity of the array. We took bright and dark flatfields for each filter with the same integration time; these were then combined and subtracted to remove the effects of dark current, telescope and dome.

The calibration was done by using the UKIRT Faint Standard Stars (Casali \& Hawarden 1992) fs18, fs23, fs24, fs 27 and fs28. We took 4 blocks of 15 images each, for every filter and for every star, at least three times per night, for different air masses. After calibration the isophote contour maps for the four observed galaxies (see Fig. 1) were obtained by means of IRAF Newcont.

\section{The deprojection method}

We divide the disk into rings with constant $\Delta R$ and assume a constant emissivity within a ring, i.e. a constant emission per unit volume in the direction of the observer $l_{i}$ (see Fig. 2). From the edge-on surface brightness, $I$, we must deduce $l_{i}$, taking into account that many rings contribute to the integral $I$, being the contribution of each ring weighted by a different area. Once $l_{i}$ is obtained we must integrate in the vertical direction to determine what would be seen if the galaxy were face-on. First, in the equation

$I_{k}=2 \Sigma_{i} A_{k i} l_{i}$

we must obtain $l_{i}$ through an inverse method. The index $k$ denotes the different pixels under observation for $z=0$. The index $i$ denotes the different rings in which the galactic plane is divided. $A_{k i}$ are the areas shown in Fig. 2.

If $k>0$

$$
\begin{aligned}
A_{k i}= & \int_{(k-1 / 2) \Delta}^{(k+1 / 2) \Delta}\left((i+1 / 2)^{2} \Delta^{2}-x^{2}\right)^{1 / 2} \mathrm{~d} x \\
& -\int_{(k-1 / 2) \Delta}^{(k+1 / 2) \Delta}\left((i-1 / 2)^{2} \Delta^{2}-x^{2}\right)^{1 / 2} \mathrm{~d} x
\end{aligned}
$$

where $x$ is an integration variable, $\Delta$ is the pixel size.

The second integral must not be calculated for $i=k$; this is the meaning of the symbol placed below the integral. In all cases, we must have $i \geq k$.

$$
\text { If } k=0
$$

$$
\begin{aligned}
A_{0 i}= & 2 \int_{0}^{\Delta / 2}\left((i+1 / 2)^{2} \Delta^{2}-x^{2}\right)^{1 / 2} \mathrm{~d} x \\
& -2 \int_{0}^{\Delta / 2}\left((i-1 / 2)^{2} \Delta^{2}-x^{2}\right)^{1 / 2} \mathrm{~d} x .
\end{aligned}
$$

The second integral must not be calculated for $i=0$. As an example,

$$
\begin{aligned}
A_{00} & =2 \int_{0}^{\Delta / 2}\left(\frac{\Delta^{2}}{4}-x^{2}\right)^{1 / 2} \mathrm{~d} x \\
& =\left[x\left(\frac{\Delta^{2}}{4}-x^{2}\right)^{1 / 2}+\frac{\Delta^{2}}{4} \sin ^{-1} \frac{x}{\Delta / 2}\right]_{0}^{\Delta / 2} \\
& =\frac{\Delta^{2}}{4} \sin ^{-1} \frac{\Delta / 2}{\Delta / 2}=\frac{\Delta^{2}}{4} \sin ^{-1} 1=\frac{\Delta^{2}}{4} \frac{\pi}{2} .
\end{aligned}
$$

In fact, this is a semicircle of radius $\Delta / 2$, with area $\frac{1}{2 \pi}(\Delta / 2)^{2}$.

In general, to calculate the above integrals we take into account that:

$$
\int\left(a^{2}-x^{2}\right)^{1 / 2} \mathrm{~d} x=\frac{1}{2}\left[x\left(a^{2}-x^{2}\right)^{1 / 2}+a^{2} \sin ^{-1} \frac{x}{|a|}\right] \text {. }
$$

We thus obtain

$$
\begin{aligned}
2 A_{k i}= & \Delta^{2}\left(\left(k+\frac{1}{2}\right)\left(\left(i+\frac{1}{2}\right)^{2}-\left(k+\frac{1}{2}\right)^{2}\right)^{1 / 2}\right. \\
& -\left(k-\frac{1}{2}\right)\left(\left(i+\frac{1}{2}\right)^{2}-\left(k-\frac{1}{2}\right)^{2}\right)^{1 / 2} \\
& -\frac{\left(k+\frac{1}{2}\right)\left(\left(i-\frac{1}{2}\right)^{2}-\left(k+\frac{1}{2}\right)^{2}\right)^{1 / 2}}{} \\
& +\frac{\left(k-\frac{1}{2}\right)\left(\left(i-\frac{1}{2}\right)^{2}-\left(k-\frac{1}{2}\right)^{2}\right)^{1 / 2}}{} \\
& +\left(i+\frac{1}{2}\right)^{2} \sin ^{-1} \frac{k+1 / 2}{i+1 / 2}-\left(i+\frac{1}{2}\right)^{2} \sin ^{-1} \frac{k-1 / 2}{i+1 / 2} \\
& -\frac{\left.\left(i-\frac{1}{2}\right)^{2} \sin ^{-1} \frac{k+1 / 2}{i-1 / 2}+\left(i-\frac{1}{2}\right)^{2} \sin ^{-1} \frac{k-1 / 2}{i-1 / 2}\right)}{}
\end{aligned}
$$

Once the areas $A_{k i}$ are calculated we need only obtain the inverse matrix. But there is a much simpler procedure, beginning with the maximum $k$ pixel $(k=N)$ and going backwards until $k=0$.

For $k=N$

$I_{N}=2 A_{N N} l_{N}$

$l_{N}=I_{N} / 2 A_{N N}$.

In general

$l_{k}=\frac{I_{k}-2 \sum_{i=k+1}^{N} A_{k i} l_{i}}{2 A_{k k}}$

because to calculate $l_{k}$ we already know all $l_{m}$ for $m>k$.

If we repeat this procedure for each $z$ we obtain a matrix $l_{i z}$. The deprojection simply implies the integration of $l_{i z}$ over $z$, and hence the surface brightness if the galaxy were observed face-on, i.e. for the deprojected galaxy, $I_{i}$ would simply be

$I_{i}=\Sigma_{z} l_{i z}$.

The determination of $N$, the last measurable point at which the galaxy signal and the sky background merge, is important. Several initial trials indicated that the deprojection was not affected by the choice of $N$, with the exception of the truncation region. Even in this case, the effect was only significant when the value of $N$ was too large. We have adopted the $3 \sigma$-criterion. 


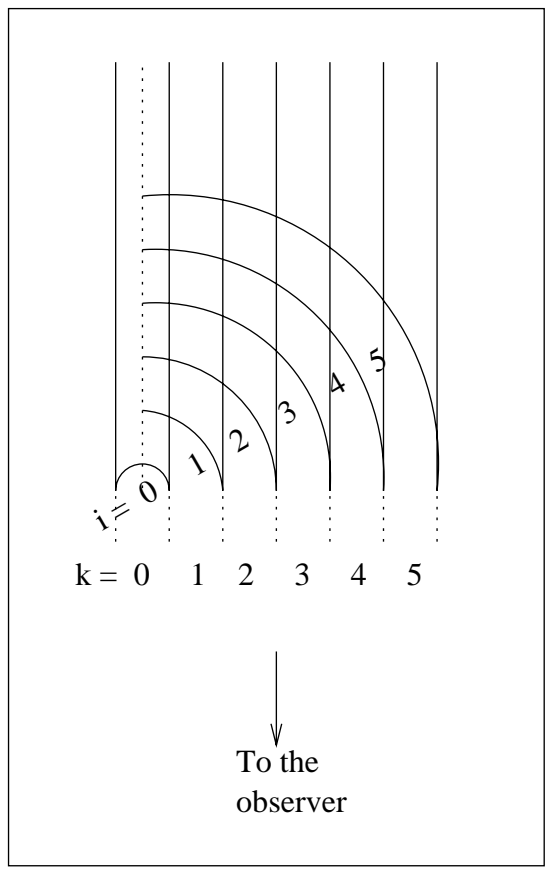

Fig. 2. Scheme for the inversion method. Subindex $i$ denotes the disk rings. Subindex $k$ denotes radial distances on the edgeon observed galaxies.

\subsection{The errors introduced by the inversion method}

Two sources of errors arise from the method itself, one of which is due to the choice of the last point considered as the transition between galaxy and background sky. As the value of the deprojected surface brightness of the more external points partly decides the values at a given position, the choice of the starting point is important. After some trials it was found that the standard $3 \sigma$-criterion was satisfactory and only unrealistic (much higher or lower) starting points produced a significantly different truncation curve.

Another source of errors arises from the formula involved, which implies a propagation of errors. The deprojected profile is slightly noisier than the observed one. Doubts could arise as to whether an error at a point, due for instance to a foreground star or just due to an error inherent in the instruments, could have a large influence at points far from the star. To evaluate the typical errors introduced by this effect, let us consider Eq. (9).

Suppose the individual errors in surface brightness, due to observation and instrumental errors, are $\Delta I_{k} \equiv \Delta I$, all approximately equal (around $2 \sigma$ ). With $A$ being a typical value of $A_{i k}$ we would have, approximately

$$
\begin{aligned}
\Delta^{2} l_{k} & =\frac{1}{(2 A)^{2}} \Delta^{2} I_{k}+\left(\frac{2 A}{2 A}\right)^{2} \sum_{i=k+1}^{N} \Delta^{2} l_{i} \\
& =\frac{1}{(2 A)^{2}} \Delta^{2} I_{k}+\left(\frac{1}{(2 A)^{2}} \Delta^{2} I_{k+1}+\ldots\right) \\
& =\frac{1}{(2 A)^{2}}(N-k) \Delta^{2} I .
\end{aligned}
$$

Therefore

$\Delta l_{k} \sim \frac{\sqrt{N-k}}{2 A} \Delta I$.
Integrating in $z$, to obtain $I_{\mathrm{d}, k}$ would therefore introduce an error of

$\Delta I_{\mathrm{d}, k}=\sqrt{\mathcal{N}_{k}} \frac{\sqrt{N-k}}{2 A} \Delta I$

where $\mathcal{N}_{k}$ is the number of layers in $z$, to be considered in this sum. Hence

$\Delta \mu=(2.5 \log e) \frac{\sqrt{\mathcal{N}_{k}} \sqrt{N-k}}{2 A} \frac{\Delta I}{I} \approx \frac{\sqrt{\mathcal{N}_{k}} \sqrt{N-k}}{2 A} \frac{\Delta I}{I}$.

For a point in the inner part of the galaxy, let us take as typical values $\mathcal{N}_{k} \sim 20 ; N-k \sim 20, A \sim 1, I \sim 300 \sigma$, $\Delta I \sim \sigma$. Then, $\Delta \mu$ is obtained to be of the order of 0.03 magnitudes.

For a point in the truncation region, $\mathcal{N}_{k} \sim 4 ; N-k \sim 4$, $A \sim 5, I \sim 10 \sigma, \Delta I \sim \sigma$. Then, $\Delta \mu \sim 0.04$ mag.

For the last adopted point, $\mathcal{N}_{k} \sim 1 ; N-k \sim 1, A \sim 10$, $I \sim 3 \sigma, \Delta I \sim \sigma$, we again obtain $0.03 \mathrm{mag}$.

We conclude that errors due to the method are of the order of 0.03 mag and that this error is constant throughout the disk. At the rim, the large relative errors in brightness are compensated by a larger typical area, $A$, as well as by the small numbers of $z$-layers available. Errors in the method itself are therefore less than $0.1 \mathrm{mag}$.

\section{Results}

In order to apply this inversion method, we first rotated all galaxies. The observed (projected) radial profiles were obtained for the interval $z=0 \pm \Delta z$, where $\Delta z$ was small ( $\approx 10-30$ arcsec), and different for each galaxy. We used data only up to the radius where the surface brightness was equal to $3 \sigma$. Particular care was taken when relatively bright stars were at the rim of the galaxy.

We then applied the model for each colour, each side and each galaxy. The quantity $l_{i z}$ was obtained, and then the intensity $I_{i}$. Results are shown in Fig. 3.

The bulge, the quasi-exponential disk and in some cases the ring, are clearly visible. What is remarkable is the truncation, which is readily observed. The truncation of all galaxies is defined in such detail that the truncation region can be studied with unprecedented precision.

Note that a large portion of the curves in Fig. 3 corresponds to points below or far below the $3 \sigma$-level given in Table 1 . The $3 \sigma$ criterion was applied to the "observed" points, but the profiles in Fig. 3 show "calculated" points. Due to the deprojection method we are deducing values of the deprojected surface brightness that would be unobservable if the galaxy were face-on, and which are therefore below the observational $3 \sigma$-level. This fact implies that the truncation phenomenon is undetectable in face-on galaxies, at least with the noise level of our observations. Also note that the $3 \sigma$ level is within the $R$-range used to fit the exponential disks. But again we must take into 

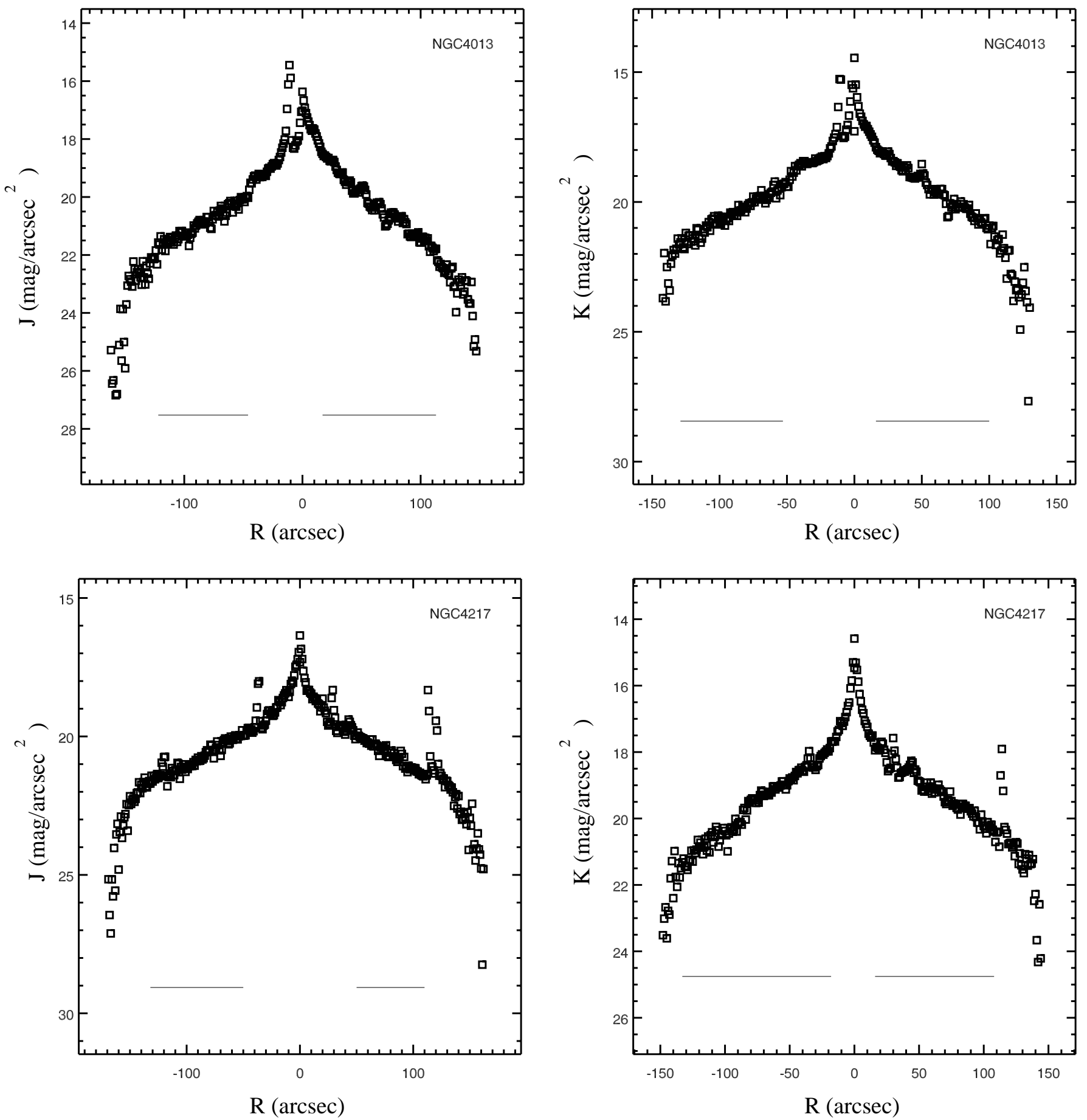

Fig. 3. Profiles obtained with the inversion method. Negative values in $R$ axis denote eastern side of the galaxies. Horizontal bars indicate the region in which the radial scale length was calculated.

account the difference between observed and deprojected points. Observed surface brightness should be obtained as an integration of deprojected surface brightness, and are therefore much higher.

\subsection{The truncation curve}

First, we obtained the radial scale length by excluding points belonging either to the bulge or to the truncation region, or even some parts of the galaxy where the radial variation was not a clear exponential function (see the horizontal lines in Fig. 3). Assuming a law of the type $I=I_{0} \mathrm{e}^{-R / R_{\mathrm{d}}}$ we obtained

$\mu=\mu_{0}+\frac{1.086}{R_{\mathrm{d}}} R$

where $\mu$ and $\mu_{0}$ are magnitudes per squared arc-second, $\mu_{0}=\mu(R=0), R$ is the galactocentric radius and $R_{\mathrm{d}}$ the 

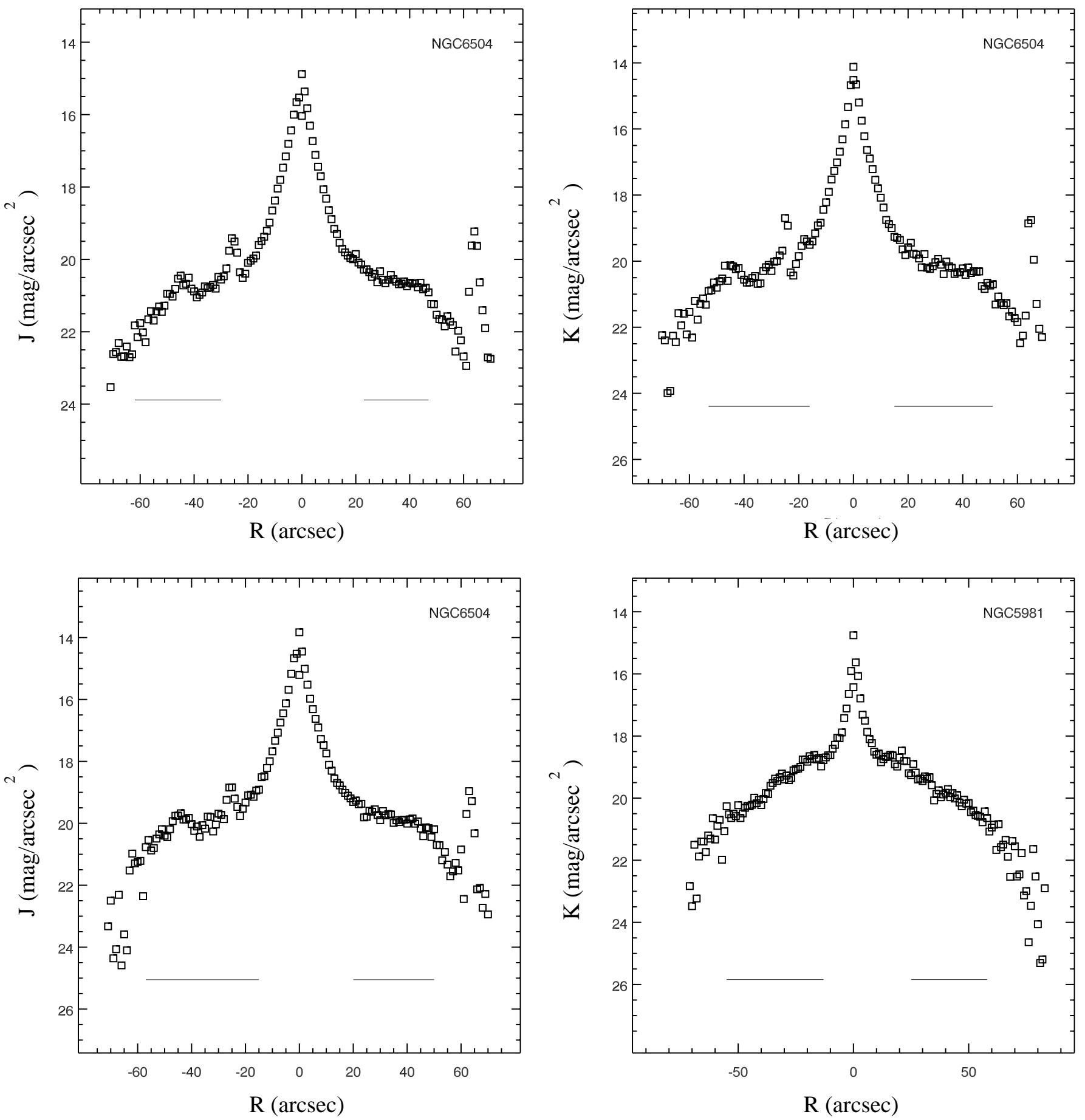

Fig. 3. continued.

radial scale length. Results are summarized in Table 3. Our results can be compared with those obtained by van der Kruit \& Searle (1982) for those galaxies present in both their and our sample. They found $R_{\mathrm{d}}=49.45^{\prime \prime}$ versus our value of $48.38^{\prime \prime}$ in $J$ and $37.83^{\prime \prime}$ in $K$ s for NGC 4217. In the case of NGC 4013 they obtained $40.34^{\prime \prime}$ whereas our data give $40.06^{\prime \prime}$ in $J$ and $33.52^{\prime \prime}$ in $K$ s. A change in scalelength between the visual and NIR means that the colour of the galaxy changes with $R$, either because of a true variation of the stellar population or to a variation due to extinction.
In the definition of the truncation curve

$T(R)=\mu(R)-\mu_{\mathrm{D}}(R)$

$\mu_{\mathrm{D}}(R)$ is equal to $\mu_{0}+\left(1.086 / R_{\mathrm{d}}\right) R$, i.e. the function $\mu(R)$ if it were a pure exponential. Plots of $T(R)$, the truncation curve, are the main objectives of this research, and are given in Fig. 4. Clearly, points of $T(R)$ in the bulge region are meaningless. 

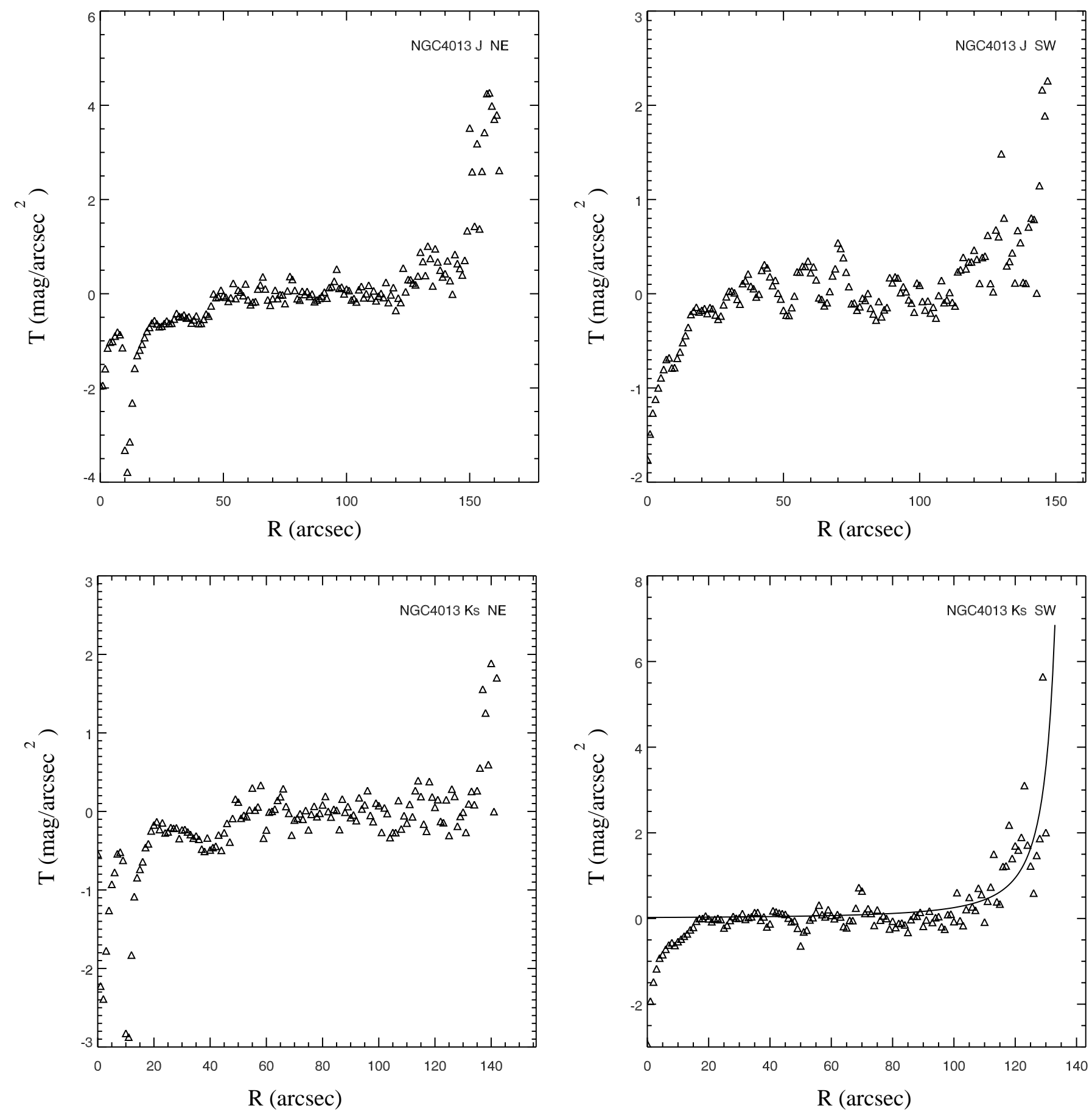

Fig. 4. Truncation curves. In the case NGC 4013, SW side, $K$ s filter, the fitted profile has been included, as an example.

In order to fit these curves, we have considered the three-parameter function

$T=\frac{a}{\left(R_{\mathrm{t}}-R\right)^{n}}$

where $a$ and $n$ are fitting parameters, as well as $R_{\mathrm{t}}$, the truncation radius, because for $R=R_{\mathrm{t}}$ we obtain $T=\infty$, so there is a complete truncation for $R=R_{\mathrm{t}}$. This function has been selected because $T$ can be very small for the large inner range where $R \ll R_{\mathrm{t}}$, but becomes larger for $R$ close to $R_{\mathrm{t}}$. Clearly this function cannot be applied for $R>R_{\mathrm{t}}$.
The fit is outlined in Table 4 . In the particular case of the NW side of NGC 6504, a bright field star is located in the truncation region. We have made different calculations with and without the star, starting before or beyond it, but the results did not differ significantly. Nevertheless, our plot for the NW side of NGC 6504 should be interpreted with extreme caution.

Values of $a$ have a large scatter. This parameter is related to the brightness close to the cutoff radius, compared with the $3 \sigma$ level. More attention should be paid to the value of the parameter $n$, as it is an exponent, therefore 

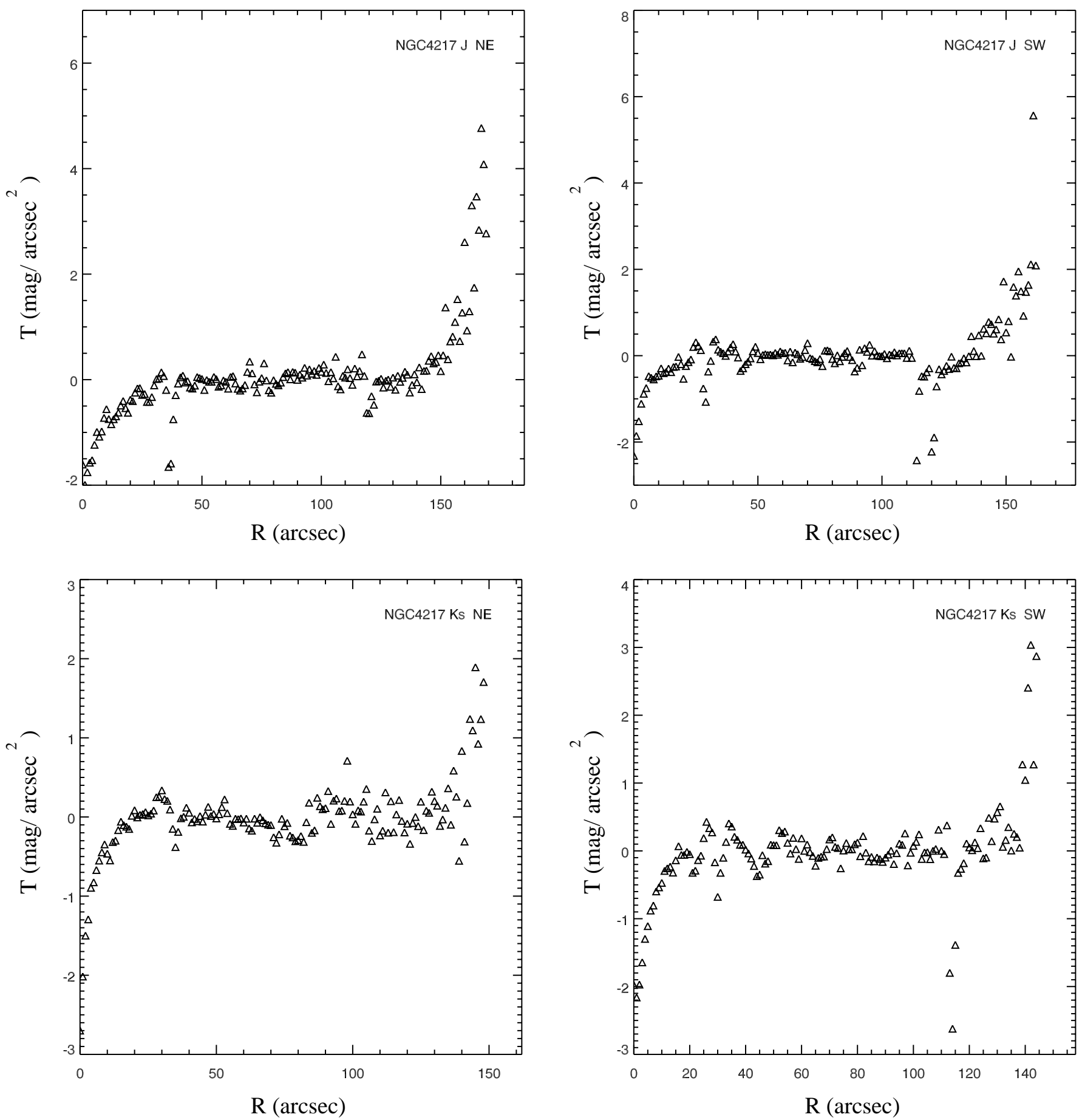

Fig. 4. continued.

characterizing the function by which the stellar population abruptly ceases. We obtain a mean value of $1.24 \sim 5 / 4$, including all filters, sides and galaxies. The mean value for the $K$ s filter is probably more representative as it is more free from extinction distortions and more accurately accounts for the stellar population. But in this case the result is remarkably similar: 1.25 with a rms of about 0.4 . The closer integer (unity) fully lies in the range of acceptable values.

The average truncation radii show a large dispersion for the different galaxies. For the average $K \mathrm{~s}$ values we obtain: NGC 4013, $R_{\mathrm{t}}=7.76 \mathrm{kpc}$; NGC 4217 , $R_{\mathrm{t}}=10.02 \mathrm{kpc} ;$ NGC 6504, $R_{\mathrm{t}}=22.97 \mathrm{kpc} ;$ NGC 5981, $R_{\mathrm{t}}=12.42 \mathrm{kpc}$. Probably the truncation would be sharper when observed with a better seeing. However no relation was found between derived truncation parameters and seeing, and therefore this effect does not significantly modify our results. 

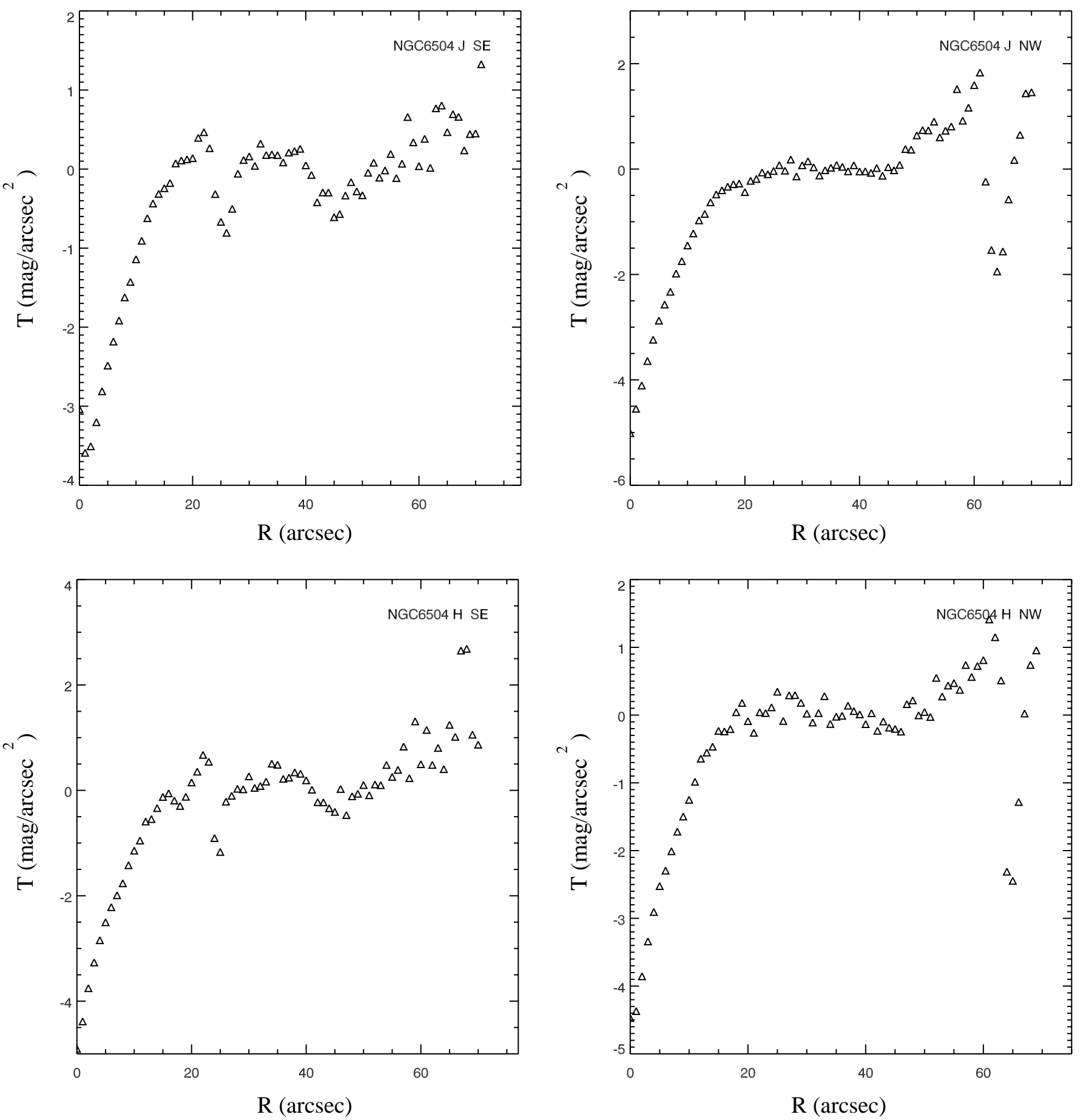

Fig. 4. continued.

\section{Discussion}

A great deal of attention has been paid previously to the coefficient $R_{\mathrm{t}} / R_{\mathrm{d}}$. In the early work by van der Kruit \& Searle (1982) it was estimated to be about $4.2 \pm 0.5$. De Grijs et al. (2000) also give values $(4.3 \pm 0.3,3.8 \pm 0.5$, $4.5 \pm 0.4$ and $2.4 \pm 0.3)$ for the four galaxies in their sample. Pohlen et al. (2000a,b) found much lower values, around $2.9 \pm 0.7$ and Barteldrees \& Dettmar (1994) give a mean value of $3.7 \pm 1.0$. In this work, where extinction and inclination effects have almost been eliminated, we also deduce lower values than van der Kruit \& Searle (1982), with 3.2 being the mean value for all the profiles considered. If we limit ourselves to the values for the longest wavelength, i.e. $K \mathrm{~s}$, we obtain for the four galaxies: NGC 4013, 4.31; NGC 4217, 4.03; NGC 6504, 2.27; NGC 5981, 3.62 (mean value of the two sides of the galaxy). The mean value for $K \mathrm{~s}$ is $3.6 \pm 0.8$. Given the high $\sigma$, our value is compatible with all previous results. Clearly, a 4-galaxy sample is too small, and more information including more types 

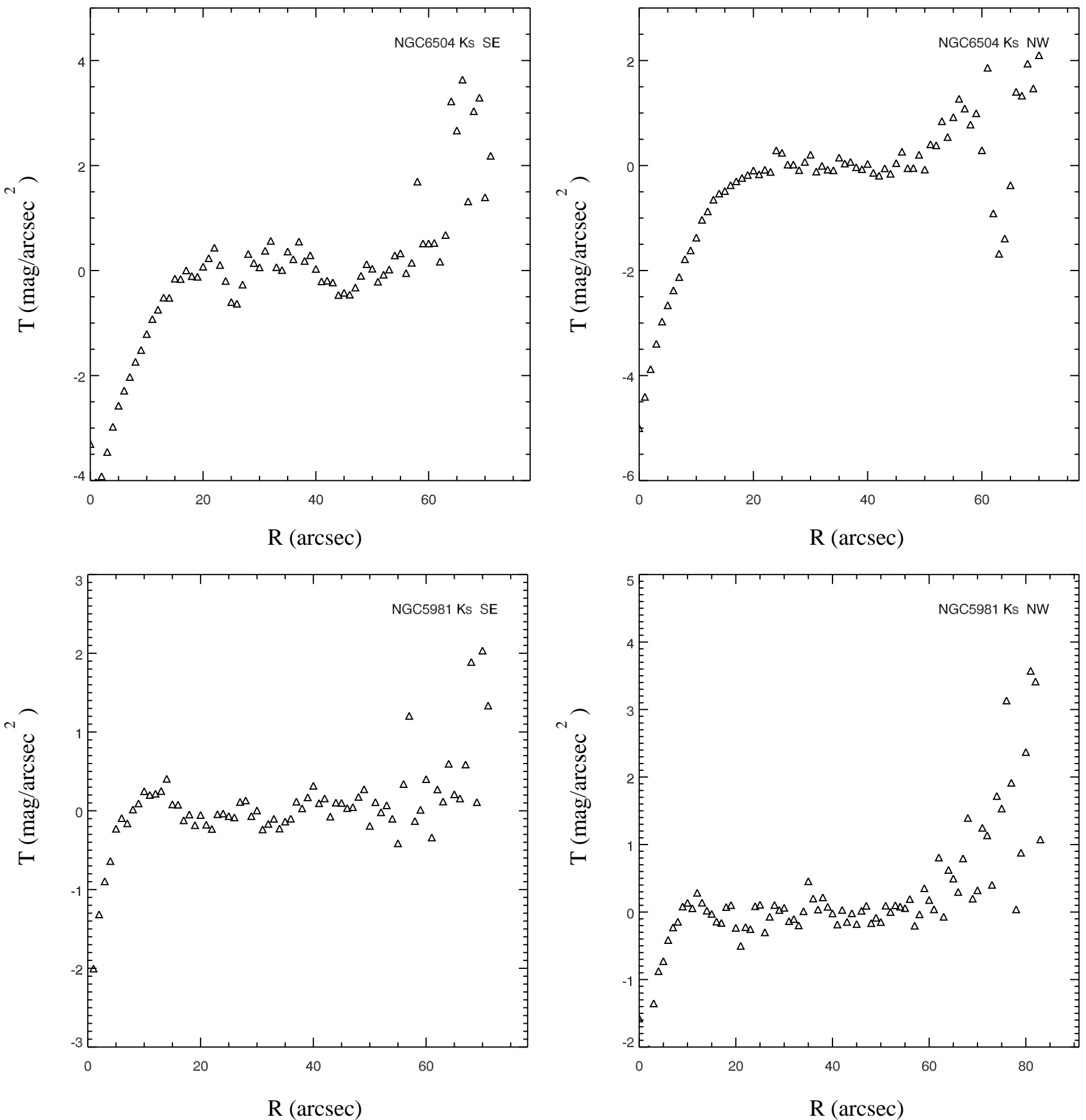

Fig. 4. continued.

of galaxies is necessary. But the data for our galaxies fit very well in Fig. 2 in Pohlen et al. (2000). So, we agree with their statement that large disks with regard to their scalelengths $R_{\mathrm{d}}$ are short in terms of their cut-off radii.

Because the extinction was assumed to be negligible in our NIR data, we cannot draw any conclusions about it. But, we consider that the $K$ s data give the most reliable results.

In general, extinction would produce an apparent higher value of the radial scale length, while $R_{\mathrm{t}}$ would be less affected as truncation takes place in regions where extinction is less important. Therefore it is believed that the optical values for $R_{\mathrm{t}} / R_{\mathrm{d}}$ are underestimated.

The difference would not be excessive, as we obtain lower but comparable values for the two galaxies we have in common with van der Kruit and Searle. The slope of the points in Fig. 5 gives the $R_{\mathrm{t}} / R_{\mathrm{d}}$ ratio. In Fig. 6 we observe a sharp correlation between $R_{\mathrm{t}}$ and the absolute magnitude of the galaxies. The brighter the galaxy, the larger the disk is found to be. This is not unexpected, but 
Table 3. Adjusted parameters for the disk.

\begin{tabular}{|c|c|c|c|c|c|}
\hline Galaxy & Passband & Side & $\begin{array}{c}R_{\mathrm{d}} \\
(\operatorname{arcsec})\end{array}$ & $\begin{array}{c}R_{\mathrm{d}} \\
(\mathrm{kpc})\end{array}$ & $\begin{array}{c}\mu_{\mathrm{o}} \\
\left(\mathrm{mag} / \operatorname{arcsec}^{2}\right)\end{array}$ \\
\hline \multirow[t]{4}{*}{ NGC 4013} & $J$ & $\mathrm{NE}$ & 48 & 2.58 & 18.98 \\
\hline & & SW & 32 & 1.75 & 18.12 \\
\hline & $K \mathrm{~s}$ & $\mathrm{NE}$ & 37 & 1.99 & 17.82 \\
\hline & & SW & 30 & 1.63 & 17.38 \\
\hline \multirow[t]{4}{*}{ NGC 4217} & $J$ & NE & 53 & 3.51 & 18.93 \\
\hline & & SW & 44 & 2.89 & 18.67 \\
\hline & $K \mathrm{~s}$ & $\mathrm{NE}$ & 35 & 2.35 & 17.28 \\
\hline & & SW & 40 & 2.65 & 17.44 \\
\hline \multirow[t]{2}{*}{ NGC 5981} & $K \mathrm{~s}$ & SE & 21 & 3.10 & 17.86 \\
\hline & & NW & 24 & 3.45 & 18.01 \\
\hline \multirow[t]{6}{*}{ NGC 6504} & $J$ & $\mathrm{SE}$ & 25 & 7.54 & 19.07 \\
\hline & & NW & 54 & 16.39 & 19.89 \\
\hline & $K \mathrm{~s}$ & $\mathrm{SE}$ & 29 & 8.99 & 18.51 \\
\hline & & NW & 38 & 11.57 & 18.83 \\
\hline & $H$ & SE & 32 & 9.91 & 19.02 \\
\hline & & NW & 32 & 9.75 & 18.99 \\
\hline
\end{tabular}

Table 4. Fit parameters for the truncation curve.

\begin{tabular}{|c|c|c|c|c|c|c|c|}
\hline Galaxy & Pas: & Side & $\begin{array}{c}R_{\mathrm{t}} \\
\mathrm{rcse}\end{array}$ & $\begin{array}{c}R_{\mathrm{t}} \\
(\mathrm{kpc})\end{array}$ & $a$ & $n$ & $\overline{R_{\mathrm{t}} / R_{\mathrm{d}}}$ \\
\hline \begin{tabular}{|ll} 
NGC 4013 \\
\end{tabular} & & $\mathrm{NE}$ & 160 & 8.65 & 2.43 & 1.10 & 3.34 \\
\hline & & SW & 155 & 8.38 & 3.56 & 0.77 & 4.79 \\
\hline & $K \mathrm{~s}$ & $\mathrm{NE}$ & 147 & 7.95 & 5.64 & 1.04 & 3.98 \\
\hline & & SW & 140 & 7.57 & 281.46 & 1.90 & 4.64 \\
\hline NGC 4217 & & $\mathrm{NE}$ & 170 & 11.24 & 11.59 & 1.15 & 3.20 \\
\hline & & SW & 165 & 10.91 & 12.30 & 1.12 & 3.78 \\
\hline & $K \mathrm{~s}$ & $\mathrm{NE}$ & 155 & 10.25 & 7.10 & 1.00 & 4.36 \\
\hline & & SW & 148 & 9.79 & 1.07 & 0.59 & 3.69 \\
\hline NGC 5981 & $K \mathrm{~s}$ & $\mathrm{SE}$ & 73 & 10.68 & 1.27 & 0.68 & 3.44 \\
\hline & & NW & 90 & 13.17 & 55.70 & 1.62 & 3.81 \\
\hline NGC 6504 & & $\mathrm{SE}$ & 75 & 22.97 & 1.15 & 0.55 & 3.05 \\
\hline & & NW & 70 & 21.44 & 665 & 2.57 & 1.29 \\
\hline & $K \mathrm{~s}$ & $\mathrm{SE}$ & 75 & 22.97 & 28.79 & 1.48 & 2.56 \\
\hline & & NW & 75 & 22.97 & 68.72 & 1.73 & 1.98 \\
\hline & $H$ & $\mathrm{SE}$ & 75 & 22.97 & 10.48 & 1.14 & 2.32 \\
\hline & & NW & 70 & 21.44 & 16.94 & 1.43 & 2.20 \\
\hline
\end{tabular}

the correlation seems to be tight. Figure 7 shows that $n$ is not clearly dependent on a typical parameter of a spiral, such as $R_{\mathrm{d}}$.

We find some colour dependence in the truncation radii. This is quite clear for NGC $4217\left(151^{\prime \prime}\right.$ in $K \mathrm{~s}, 167^{\prime \prime}$ in $J$; this tendency is confirmed by its value in the optical given by van der Kruit \& Searle (1982) of $\left.202^{\prime \prime}\right)$. It is also clear for NGC $4013\left(143^{\prime \prime}\right.$ in $K \mathrm{~s}, 157^{\prime \prime}$ in $J$; this tendency is confirmed by its value in the optical of $165^{\prime \prime}$ given by van der Kruit \& Searle). However, NGC 6504 does not show this colour dependence, $R_{\mathrm{t}}$ being nearly independent of wavelength. We have only one filter for NGC 5981. We

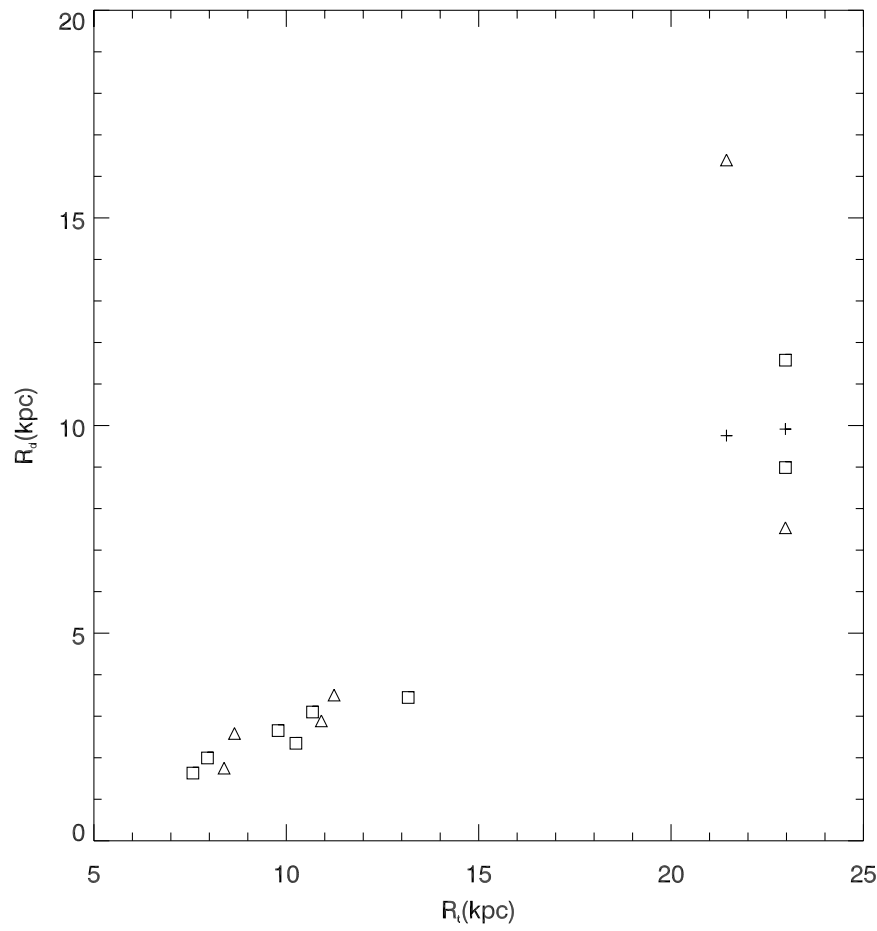

Fig. 5. $R_{\mathrm{d}}$ versus $R_{\mathrm{t}}$. Squares, $K \mathrm{~s}$ filter; triangles, $J$-filter and crosses, $H$-filter.

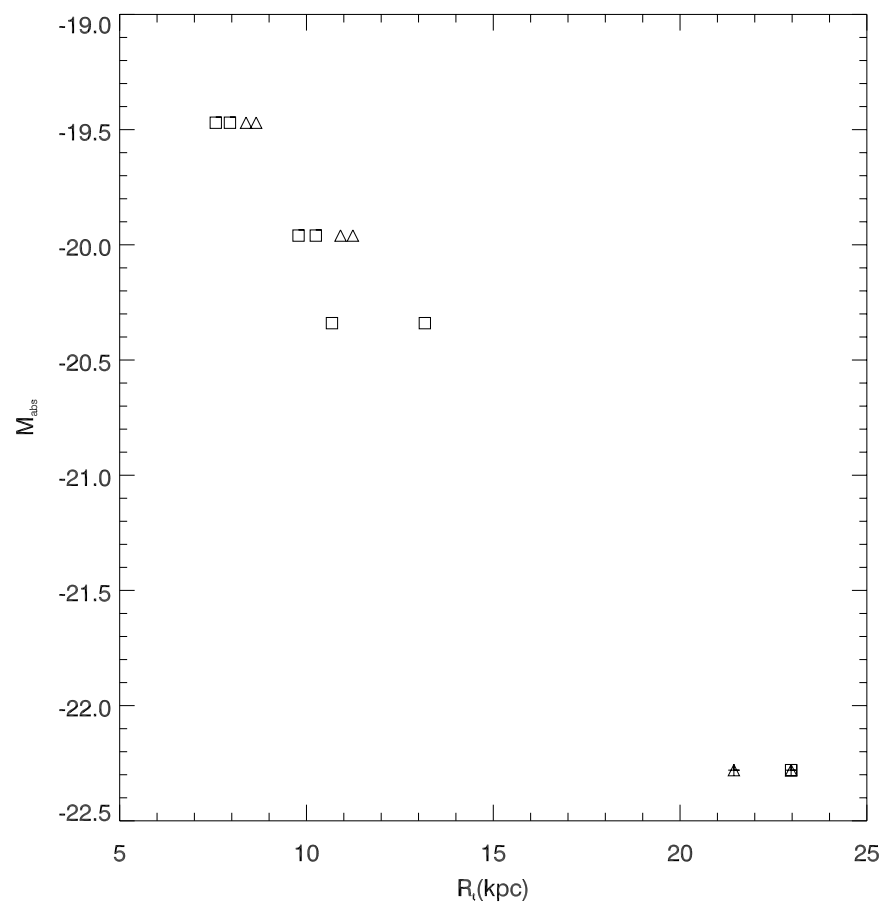

Fig. 6. Absolute $M$-magnitude versus $R_{\mathrm{t}}$. Symbols as in Fig. 5 .

suggest that $R_{\mathrm{t}}$ could be lower for the older population, but this conclusion is not at all firm. This fact could indicate that the stellar disk has grown, but the observational evidence is scarce to ascertain any interpretation of this colour dependence. 


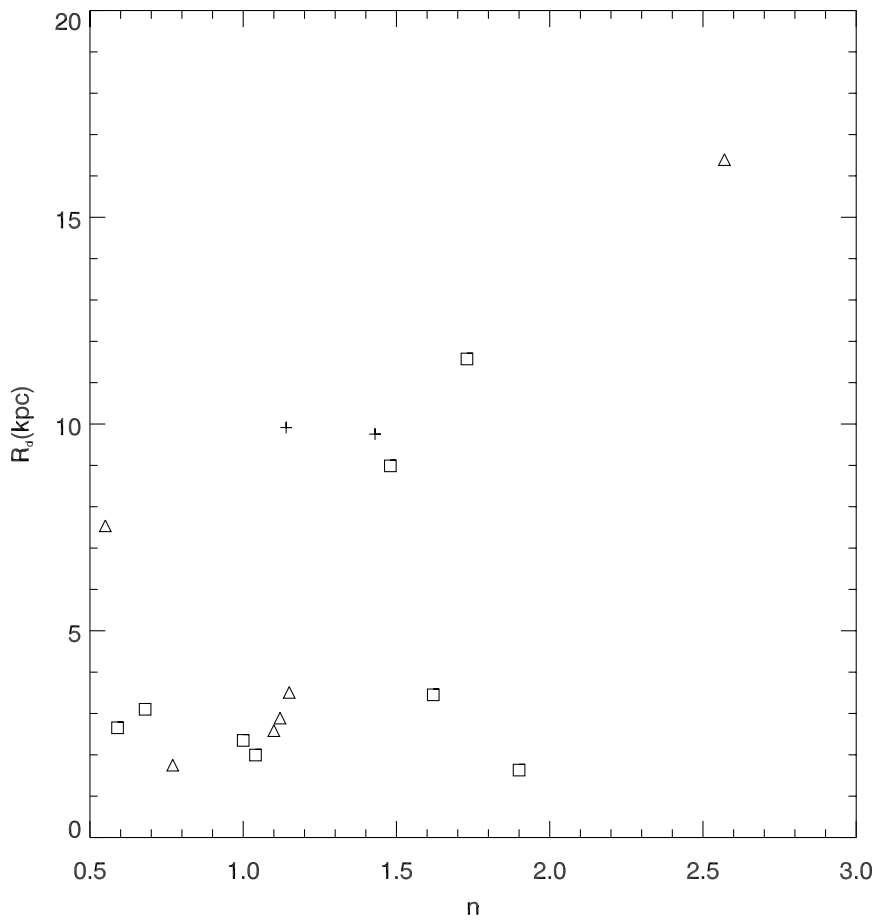

Fig. 7. $R_{\mathrm{d}}$ versus $n$. Symbols as in Fig. 5 .

The coefficient $n$ has no clear relation with other basic parameters of the galaxies. In particular no relation with colour was found. We have obtained 1.25 , with the simplest possible value (unity) compatible within the error range. The simplest truncation curve compatible with our data would therefore be

$T \propto \frac{1}{R_{\mathrm{t}}-R}$.

This is an important result. As the truncation is not sharp (e.g. de Grijs et al. 2000) the shape of the truncation curve should be investigated by theoretical methods, and compared with Eq. (18). The theoretical prediction of this truncation curve would be more restrictive than that of any other observable parameters.

Asymmetries have been observed but these are not large. The parameter

$2 \frac{\left|\left(R_{\mathrm{t}}(\mathrm{E})-R_{\mathrm{t}}(\mathrm{W})\right)\right|}{R_{\mathrm{t}}(\mathrm{E})+R_{\mathrm{t}}(\mathrm{W})}$

(where $\mathrm{E}$ and $\mathrm{W}$ denote the two sides of the galaxies) characterizing the relative degree of asymmetry was only 0.05 , 0.05, 0.00 and 0.21, for NGC 4013, NGC 4217, NGC 6504 and NGC 5981 respectively, if we only take into account the more confident values of $K \mathrm{~s}$.

A potentially very restrictive fact suggested by van der Kruit (2000), that the rotation curve has a decreasing step just after the truncation, can neither be rejected nor confirmed as the rotation curve of NGC 4013 (Verheijen 1997) shows this step $4 \mathrm{kpc}$ after $R_{\mathrm{t}}$, but it is apparently not present for NGC 4217 (see also Verheijen 1997).

\section{Conclusions}

The stellar disk is less extended than the gaseous disk and this is not the result of sensitivity limits of optical telescopes: there is a physical mechanism that produces a relatively sharp truncation. To investigate this mechanism, the advantages of observing in the near infrared are obvious as we are looking for a phenomenon concerning the stars, and because the results are not as affected by extinction problems, whose radial distribution is not "a priori" known. The advantages of an inversion method not prescribing ad-hoc mathematical models for the different galactic components have been shown. In particular, the use of a model with several free parameters for the truncation region prevents us from obtaining the function we seek, i.e. the detailed way in which the stellar component leaves the exponential distribution and becomes completely truncated at $R_{\mathrm{t}}$.

To investigate the mechanism responsible for the truncation of the stellar disk, more observational effort is needed, especially in the near infrared. Also, more theoretical work is needed as many of the hypotheses advanced up to now fail to explain firm constraints that have been established by observations.

The possible relation between $R_{\mathrm{t}}$ and warps, which was considered by van der Kruit (2000) is not clear: for NGC 4013 and NGC 6504, we can see in Florido et al. (1991) that the warps begin at a radius that is much smaller than $R_{\mathrm{t}}$.

Two important conclusions can be extracted from this work:

- The coefficient $R_{\mathrm{t}} / R_{\mathrm{d}}$ has been found to be lower than in some previous works, being for $K \mathrm{~s} 3.6 \pm 0.8$, at least for the four galaxies observed;

- We suggest that a colour dependence of the truncation radius exists: truncation seems to take place at lower radii for larger wavelengths, but this was only for $2 / 3$ of the small sample. More observations are needed.

We present plots of the truncation curve $T(R)$, which is one of the main objectives of this work. These plots are more informative than the truncation radius itself. Nevertheless, we have tried to fit these $T(R)$ curves. A reasonable candidate formula is of the type $T(R) \propto$ $\left(R_{\mathrm{t}}-R\right)^{-n}$, where the exponent $n$ has been found to be $1.25 \pm 0.4$. This suggest $n=5 / 4$, with unity being a value within the errors. A simple suggestion is therefore

$T(R) \propto 1 /\left(R_{\mathrm{t}}-R\right)$.

Acknowledgements. We are thankful to Dr. Peletier for providing his very efficient reduction software REDUCE and for many helpful discussions during the period of this work.

The CST is operated on the island of Tenerife by the Instituto de Astrofísica de Canarias in the Spanish Observatorio del Teide of the Instituto de Astrofísica de Canarias.

This paper has been supported by the "Plan Andaluz de Investigacion" (FQM-108) and by the "Secretaría de Estado de Política Científica y Tecnológica" (AYA2000-1574). 


\section{References}

Barteldrees, A., \& Dettmar, R. J. 1994, A\&AS, 103, 475

Binney, J., \& Tremaine, S. 1987, Galactic Dynamics (Princeton University Press)

Brand, J., \& Wouterloot, J. G. A. 1991, in The interstellar disk-halo connection in Galaxies, ed. H. Bloemen, 121

Brand, J., \& Wouterloot, J. G. A. 1994, A\&AS, 103, 503

Brand, J., \& Wouterloot, J. G. A. 1995, A\&A, 303, 851

Brand, J., \& Wouterloot, J. G. A. 1996, in Unsolved problems of the Milky Way, ed. L. Blitz \& P. Teuben, 561

Casali, M. M., \& Hawarden, T. G. 1992, JCMT-UKIRT Newslett., 3, 33

Ferguson, A. M., Gallagher, J. S., \& Wyse, R. F. G. 1998, AJ, 116,673

Florido, E., Prieto, M., Battaner, E., Mediavilla, E., \& Sanchez-Saavedra, M. L. 1991, A\&A, 242, 301

Freudenreich, H. T. 1998, ApJ, 492, 495

de Grijs, R., Kregel, M., \& Wesson, K. H. 2001 [astro-ph/0002523]

Gunn, J. E. 1982, in Astrophysical Cosmology, Vatican City State, 233

Habing, H. J. 1988, A\&A, 200, 40

Kennicutt, R. C. 1989, ApJ, 344, 685

Kobayashi, N., \& Tokunaga, A. T. 2000, ApJ, 532, 423

van der Kruit, P. C. 1979, A\&AS, 38, 15

van der Kruit, P. C. 2001, in Galaxy Disks and Disk Galaxies, ed. J. G. Funes, \& E. M. Corsini, ASP Conf. Ser.

van der Kruit, P. C., \& Searle, L. 1981a, A\&A, 95, 105

van der Kruit, P. C., \& Searle, L. 1981b, A\&A, 95, 116

van der Kruit, P. C., \& Searle, L. 1982, A\&A, 110, 61
Larson, R. B. 1976, MNRAS, 176, 31

Lequeux, J., \& Guélin, M. 1996, in New Extragalactic Perspectives, The New South Africa, ed. D. Block

May, J., Alvarez, H., \& Bronfman, L. 1997, A\&A, 327, 325

Mead, K. N., \& Kutner, M. L. 1988, ApJ, 330, 399

Mead, K. N., Kutner, M. L., \& Evans, N. J. 1990, ApJ, 354, 492

Mead, K. N., Kutner, M. L., Evans, N. J., Harvey, P. M., \& Wilking, B. A. 1987, ApJ, 312, 321

Pohlen, M., Dettmar, R. J., \& Lütticke, R. 2000, A\&A, 357, L1

Pohlen, M., Dettmar, R. J., Lütticke, R., \& Schwarzkopf, U. 2000, A\&AS, 144, 405

Porcel, C., Battaner, E., \& Jiménez-Vicente, J. 1997, A\&A, 322, 103

Robin, A. C., Crézé, M., \& Mohan, V. 1992, ApJ, 400, L25

Rudolph, A. L., Brand, J., de Geus, E. J., \& Wouterloot, J. G. A. 1996, ApJ, 458, 653

Ruphy, S., Robin, A. C., Epchtein, N., et al. 1996, A\&A, 313, L21

Santos, C. A., Yun, J. L., Clemens, D. P., \& Agostinho, R. J. 2000, ApJ, 540, L87

Verheijen, M. A. W. 1997, Ph.D. Thesis, Kapteyn Institute

Williams, J. P., \& McKee, C. F. 1997, ApJ, 476, 166

Wouterloot, J. G. A., \& Brand, J. 1996, A\&AS, 119, 439

Wouterloot, J. G. A., Brand, J., \& Henkel, C. 1988, A\&A, 191, 323

Wouterloot, J. G. A., Fiegle, K., Brand, J., \& Winnewisser, G. 1995, A\&A, 301, 236

Wouterloot, J. G. A., Fiegle, K., Brand, J., \& Winnewisser, G. 1997, A\&A, 319, 360 